\title{
Curcumin alleviates brain edema by lowering AQP4 expression levels in a rat model of hypoxia-hypercapnia-induced brain damage
}

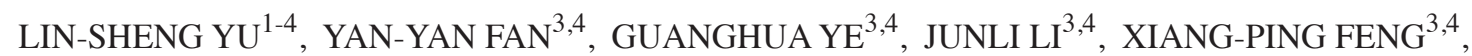 \\ KEZHI LIN ${ }^{3,4}$, MIUWU DONG ${ }^{3,4}$ and ZHENYUAN WANG ${ }^{1,2}$ \\ ${ }^{1}$ Shanghai Key Lab of Forensic Medicine, Shanghai City West, Shanghai 200063; \\ ${ }^{2}$ Xi'an Jiaotong University Health Science Center, School of Forensic Science and Medicine, Xi'an, Shaanxi 710061; \\ ${ }^{3}$ Department of Forensic Medicine; ${ }^{4}$ Institute of Forensic Science, Wenzhou Medical University, \\ Wenzhou, Zhejiang 325035, P.R. China
}

Received October 21, 2014; Accepted October 8, 2015

DOI: $10.3892 / \mathrm{etm} .2016 .3022$

\begin{abstract}
The present study aimed to investigate the therapeutic effects of curcumin (CU) against brain edema in a rat model of hypoxia-hypercapnia (HH)-induced brain damage (HHBD). Male Sprague-Dawley rats were divided into five groups, including a control group and four treatment groups. The rats in the control group were raised under normal laboratory conditions and were injected with water, whereas the rats in the treatment groups were exposed to a low $\mathrm{O}_{2} /$ high $\mathrm{CO}_{2}$ environment simulating $\mathrm{HH}$ conditions, and were injected with water, CU, dimethyl sulfoxide (solvent control) or monosialoganglioside GM1. After 2 weeks, the morphological characteristics of the brain tissues were analyzed using optical and electron microscopy. In addition, aquaporin (AQP)-4 protein expression levels in brain tissue samples were analyzed using streptavidin-biotin complex immunohistochemistry and western blotting, and mRNA expression levels were detected using reverse transcription-quantitative polymerase chain reaction. Severe brain edema, tissue structure disruption and increased AQP4 expression levels were detected in the brain tissues of the $\mathrm{HH}$ rats. Conversely, the rats treated with CU or GM1 exhibited attenuated HHBD-induced brain edema and tissue structure disruption, and decreased mRNA and protein expression levels of AQP4. The results of the present study suggested that $\mathrm{CU}$ treatment was able to attenuate HHBD-induced brain edema by downregulating the expression levels of AQP4 in a rat model. Therefore, CU may be considered a potential therapeutic drug for the treatment of patients with brain edema.
\end{abstract}

Correspondence to: Dr Zhenyuan Wang, Shanghai Key Lab of Forensic Medicine, Shanghai City West, 1347 Guangfu Road, Shanghai 200063, P.R. China

E-mail: zhenyuanwdr@163.com

Key words: chronic hypoxia-hypercapnia-induced brain damage, brain edema, aquaporin- 4 , curcumin

\section{Introduction}

Hypoxia-hypercapnia (HH)-induced brain damage (HHBD), which is commonly detected during the process of asphyxiation and chronic obstructive pulmonary disease (COPD), has previously been associated with nervous system disorders, mental retardation and cerebral palsy (1). In addition, HHBD is a type of brain injury that is associated with high rates of neonatal and adult morbidity and mortality (2). Brain edema, which is defined as an increase in brain water content, is a common pathological characteristic of HHBD (3). Brain edema is characterized by cell swelling, which alters the concentrations of cellular metabolites and subsequently disturbs normal cellular functions (4). In addition, edema may lead to rapid increases in intracranial pressure, which has previously been associated with headaches, comas and life-threatening herniation (5). Therefore, the development of effective therapeutic drugs that are able to limit the occurrence of HHBD-induced brain edemia is required.

Various neuroprotective agents have been applied for the treatment of brain edema, including oxygen free radical scavengers, calcium channel blockers, excitatory amino acid antagonists and neurotrophic factors. These drugs, which typically have a single therapeutic target and defined underlying pharmacological mechanisms, have demonstrated effectiveness in various experimental studies; however, they have shown mixed effectiveness and, in some cases serious side-effects, in human clinical trials (6-8).

Previous studies have demonstrated that various traditional Chinese medicines may exert neuroprotective effects, including the prevention of neuronal cell death and the proliferation of neural stem cells $(9,10)$. In addition, numerous herbal medicines have exhibited therapeutic effectiveness against brain edema $(11,12)$. Therefore, traditional Chinese medicines may be considered a source of potential drugs for the treatment of brain edema.

Curcumin (CU), which is a low molecular weight spice, is isolated from the rhizome of the Curcumin longa plant (13), and has been used in the past for medicinal and food-coloring purposes (14). Previous studies demonstrated that CU was able to 
attenuate neuroinflammation and neurological injury in patients with Alzheimer's disease, ischemic stroke and subarachnoid hemorrhage (15-17).

Our previous study investigated the effects of $\mathrm{CU}$ against hypoxic-ischemic brain damage (HIBD) in a rat model (18), and demonstrated that CU treatment was able to partially attenuate HIBD-induced brain edema and morphological tissue changes. These effects of CU were demonstrated to occur as a result of nitric oxide synthase activity inhibition and decreased expression levels of aquaporin (AQP)-4 in the hippocampus of the rat. The present study established a novel rat model of HHBD by exposing the rats to a low $\mathrm{O}_{2} /$ high $\mathrm{CO}_{2}$ environment, which simulated $\mathrm{HH}$ conditions. In addition, the effects of $\mathrm{CU}$ against HHBD were investigated using various techniques, including hematoxylin and eosin (HE) staining, electron microscopy, streptravidin-biotin complex (SABC) immunohistochemistry, western blotting and reverse transcription-quantitative polymerase chain reaction (RT-qPCR). In particular, the present study focused on the association between CU treatment and AQP4 expression levels in the rat model of HHBD, with the intention of elucidating the underlying mechanism by which CU may attenuate HHBD-induced brain edema.

\section{Materials and methods}

Rats and ethics statement. A total of 30 healthy male Sprague-Dawley rats (age, 6-7 weeks; weight, 200-310 g) were purchased from the Experimental Animal Center of Wenzhou Medical College (Wenzhou, China). Rats were housed under a $12 \mathrm{~h} \mathrm{light/dark}$ cycle at a controlled temperature of $22-25^{\circ} \mathrm{C}$ and a humidity of $55 \pm 5 \%$, with ad libitum access to water and food throughout the study. After acclimatization to the laboratory environment for 1-2 days, the rats were randomly divided into five groups, as follows (6 rats/group): i) Control (CK) group, in which the rats were raised under normal laboratory conditions; ii) HH group, in which the rats were exposed to $\mathrm{HH}$ conditions without drug treatment; iii) $\mathrm{CU}$ group (Sigma-Aldrich, St. Louis, MO, USA), in which the rats were exposed to $\mathrm{HH}$ conditions and were subsequently treated with CU; iv) dimethyl sulfoxide (DMSO; Sigma-Aldrich) group, in which the rats were exposed to $\mathrm{HH}$ conditions and were subsequently injected with DMSO; and v) monosialoganglioside (GM1; Sigma-Aldrich) group, in which the rats were exposed to $\mathrm{HH}$ conditions and were treated with GM1. The present study was approved by the Medical Ethics Committee of Wenzhou Medical College, and all procedures were in compliance with the National Institute of Health Guide for the Care and Use of Laboratory Animals (NIH Publications no. 80-23).

Selection of $C U$ dose. In order to determine the effective dose of CU, a range of CU doses $(0,20,40,60$ and $80 \mathrm{mg} / \mathrm{kg})$ were analyzed in a trial experiment, in which DMSO was used as the solvent, according to our previous study (18). A $40 \mathrm{mg} / \mathrm{kg} \mathrm{CU}$ dose was selected for further experiments, as doses $>40 \mathrm{mg} / \mathrm{kg}$ resulted in similar results (data not shown).

Rat model of HHBD. All rats, except those in the CK group, were maintained in an airtight container and were subjected to low levels of $\mathrm{O}_{2}(9-11 \%)$ and high levels of $\mathrm{CO}_{2}(5-6 \%)$ for
8 h every day. The chronic intermittent hypoxia treatment lasted for 2 weeks. Conversely, the rats in the CK group were maintained in an open laboratory environment. The rats in all groups were fed using an identical protocol, according to a previous report (19). Prior to the rats entering the container, equal volumes of $\mathrm{CU}(10 \mathrm{mg} / \mathrm{ml} ; 40 \mathrm{mg} / \mathrm{kg}$ for rats in the $\mathrm{CU}$ group), DMSO (for rats in the DMSO group), GM1 (10 mg/ml; $3 \mathrm{mg} / \mathrm{kg}$ for rats in the GM1 group) and water (for rats in the $\mathrm{HH}$ and $\mathrm{CU}$ groups) were injected into the abdomen of the rats.

Tissue collection and preparation. The rats were decapitated following 2 weeks of chronic intermittent hypoxia treatment, and were maintained on ice. The occiput was immediately cut open from the foramen magnum forward along the midline. After the dura and pia were stripped, the brain cortex, cerebellum and brain stem were removed completely and rinsed with pre-cooled saline water. The brain tissues $(\sim 1 / 3)$ were frozen and stored in liquid nitrogen for RT-qPCR analysis $(20,21)$, or they were used for water content analysis $(\sim 1 / 3)$. The remaining brain tissue was prepared and visualized using optical and electron microscopy, and SABC immunohistochemical analysis.

Optical microscopy analysis. Tissue samples corresponding to the brain cortex, cerebellum and brainstem were cut on a wax board and were subsequently fixed at $4^{\circ} \mathrm{C}$ with $4 \%$ paraformaldehyde. Following fixation, the tissues were washed and dehydrated with gradient alcohol, after which the tissues were cleared with xylene, immersed in paraffin and embedded. Subsequently, $4 \mu \mathrm{m}$ tissue sections were prepared for $\mathrm{HE}$ staining (Sigma-Aldrich) and immunohistochemistry. Tissues were observed under an optical microscope (Eclipse E200; Nikon Corporation, Tokyo, Japan) (22).

Electron microscopy analysis. Brain tissue samples were collected and fixed initially with $2.5 \%$ glutaraldehyde and then with $1 \%$ osmium tetroxide. After fixing, the tissues were dehydrated with gradient mixtures of ethanol and acetone and embedded in Epon 812 (Electron Microscopy Sciences, Hatfield, PA, USA). Subsequently, the tissues were cut into $70 \mathrm{~nm}$ sections, and double-stained with uranyl acetate and lead nitrate. Tissue samples were observed under a transmission electron microscope (JEM-3010; JEOL, Ltd., Tokyo, Japan) (18).

Water content measurement. The water levels in the brain tissues from the rats in the various treatment groups were measured according to a previous study (23). Briefly, brain tissue $(\sim 0.15 \mathrm{~g})$ from each rat was sliced, weighed and completely dried in an oven at $110^{\circ} \mathrm{C}$ for $24 \mathrm{~h}$. The dried tissue was weighed again, and the water percentage of the brain tissue was calculated using the following formula: Water $(\%)=[($ wet weight-dried weight)/wet weight] x $100 \%$.

SABC immunohistochemical analysis of AQP4 expression levels. AQP4 expression levels in the cortex, cerebellum, and brainstem tissue samples were analyzed using the SABC immunohistochemical method. The AQP4 antibody and the SABC kit were purchased from OriGene Technologies, Inc. (Beijing, China). The assay, image detection and analysis procedures were performed according to our previous report (18). 

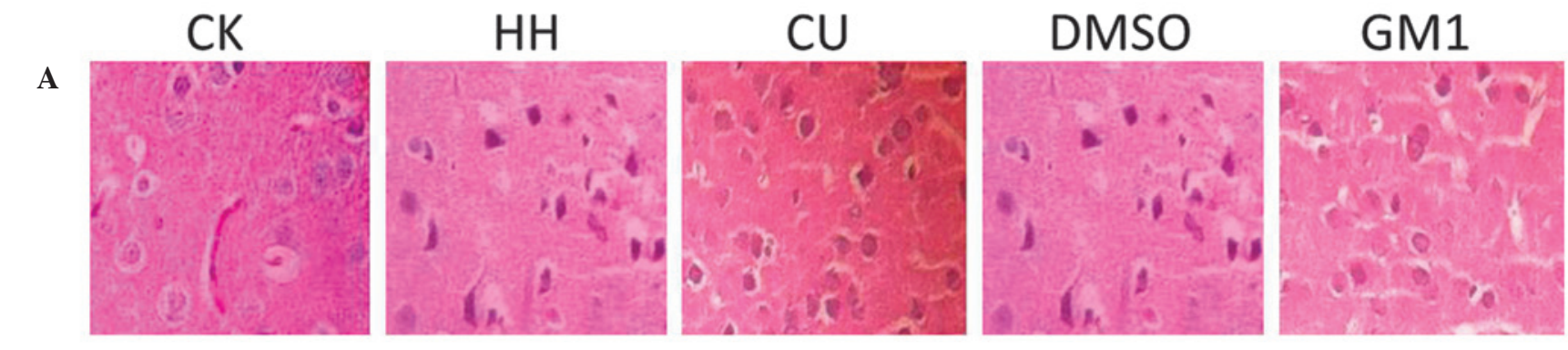

B
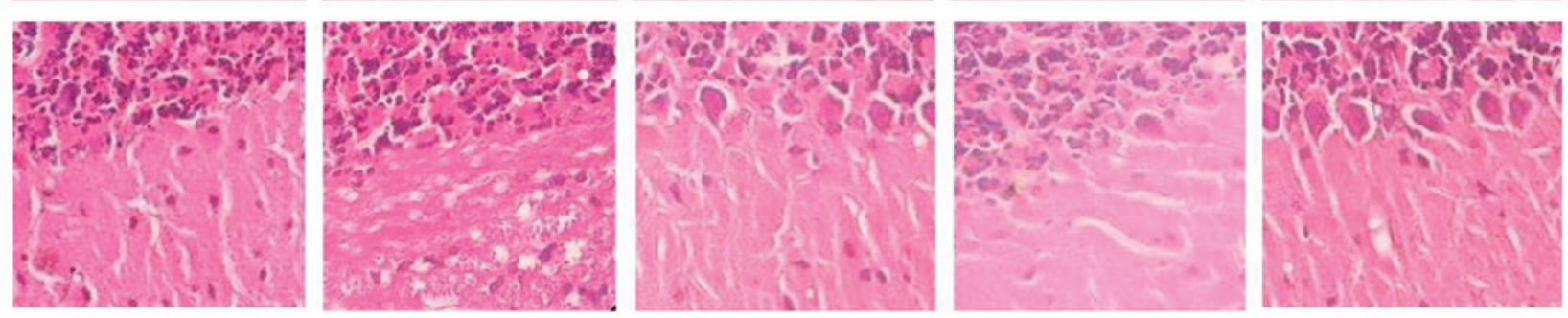

C
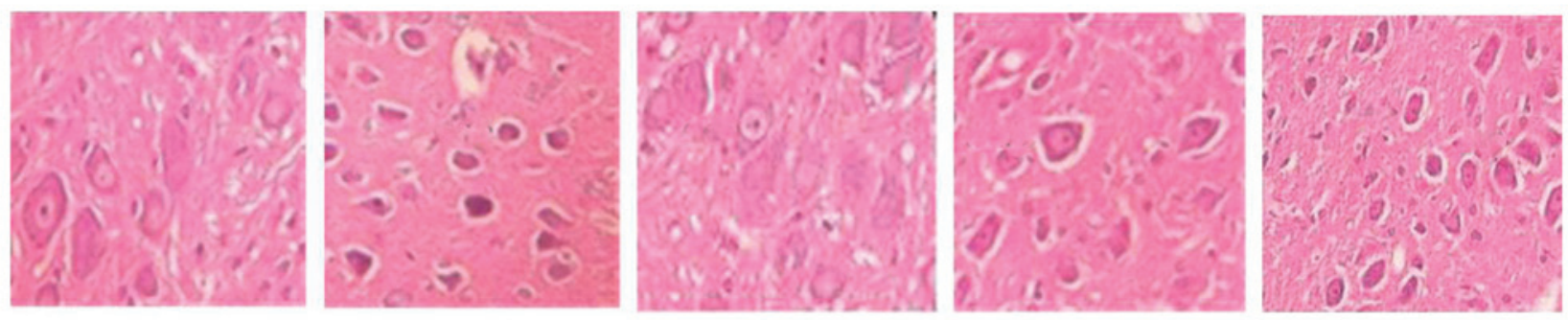

Figure 1. Hematoxylin and eosin staining and light microscopy analysis of brain tissues (magnification, $\mathrm{x} 400 ; 14$ days) derived from the (A) brain cortex, (B) cerebellum and (C) brainstem regions of rats in the various treatment groups. Fields ( $>5$ ) with positive results were identified in each case. CK, control group; $\mathrm{HH}$, hypoxia-hypercapnia group; $\mathrm{CU}$, curcumin group; DMSO, dimethyl sulfoxide solvent group; GM1, monosialoganglioside group.

Western blot analysis of AQP4 protein expression levels. AQP4 protein expression levels were also analyzed by western blotting. Briefly, total protein was extracted according to a previous study (24), and the total protein concentration was measured using the bicinchoninic acid assay (Pierce Biotechnology, Inc., Rockford, IL, USA). Subsequently, $50 \mu \mathrm{g}$ protein samples were separated by $12 \%$ sodium dodecyl sulfate-polyacrylamide gel electrophoresis and transferred to nitrocellulose membranes (Schleicher \& Schuell BioScience, Inc., Keene, NH, USA). The membranes were washed with Tris-buffered saline containing $0.1 \%$ Tween 20 (TBST; Sigma-Aldrich) and blocked with non-fat dry milk, after which they were incubated with rabbit polyclonal anti-AQP4 (1:500; TA326513, OriGene Technologies, Inc., Beijing, China) and mouse monoclonal anti-glyceraldehyde 3-phosphate dehydrogenase (GAPDH; loading control; 1:5,000; sc-365062, Kangchen Biotechnology Inc., Shanghai, China) antibodies, at $4^{\circ} \mathrm{C}$ overnight. Following repeated washes with TBST, the membranes were incubated with goat anti-mouse horseradish peroxidase-conjugated secondary antibodies $(1: 5,000$; sc-2005; Jackson Immunoresearch Laboratories, Inc., West Grove, PA, USA) for $2 \mathrm{~h}$ at room temperature. Subsequently, target bands were detected using Hyperfilm Enhanced Chemiluminescence (RPN3103 K) and X-ray exposure (GE Healthcare Life Sciences, Chalfont, UK). Optical density (OD) values were quantitatively analyzed using a laser densitometer (UltroScan XL; GE Healthcare Life Sciences), and the results of AQP4 expression were reported as percentage changes over GAPDH (25).
Total RNA extraction and RT-qPCR. In order to validate the AQP4 protein expression levels detected in the brain tissues of the $\mathrm{CK}, \mathrm{CU}, \mathrm{HH}, \mathrm{DMSO}$ and GM1 groups at the transcriptional level in vivo, RT-qPCR was performed using the StepOne ${ }^{\mathrm{TM}}$ RT-PCR system (Applied Biosystems; Thermo Fisher Scientific, Inc., Waltham, MA, USA). Total RNA was extracted from tissues, which were homogenized using an electrical homogenizer and an RNeasy Plant Mini kit (Qiagen $\mathrm{GmbH}$, Hilden, Germany), according to the manufacturer's protocol. Subsequently, extracted RNA was treated with the RNase-Free DNase kit (Qiagen $\mathrm{GmbH}$ ), after which cDNA $(1 \mu \mathrm{g})$ was obtained by reverse transcription using the PrimeScript RT-PCR kit (Takara Bio, Inc., Otsu, Japan). The primer pairs were designed for amplification of the GAPDH and AQP4 genes with the use of Premier 5.0 software (Premier Biosoft International, Palo Alto, CA, USA), and were synthesized by Shanghai Sunred Biological Technology Co., Ltd. (Shanghai, China). The sequences of the primer pairs were as follows: AQP4 (110 bp) forward, 5'-ACCCTGGACAGCTGT AAGTGTGGA-3' and reverse, 5'-AGGAACTCTGCTGTG ACCGCCT-3'; and GAPDH (93 bp) forward, 5'-GGGAAA TCGTGCGTGACATT-3' and reverse, 5'-GCGGCAGTGGCC ATCTC-3'. The cycling conditions were as follows: $10 \mathrm{~min}$ at $95^{\circ} \mathrm{C}$, followed by 40 cycles of $95^{\circ} \mathrm{C}$ for $20 \mathrm{sec}, 56^{\circ} \mathrm{C}$ for $30 \mathrm{sec}$ and $72^{\circ} \mathrm{C}$ for $30 \mathrm{sec}$, and a final extension step at $72^{\circ} \mathrm{C}$ for $10 \mathrm{~min}$. Three independent replicates were performed for each sample. The comparative quantification cycle $(\mathrm{Cq})$ method was used to determine relative levels of gene expression. The GADPH gene was used as an internal control. mRNA tran- 
A
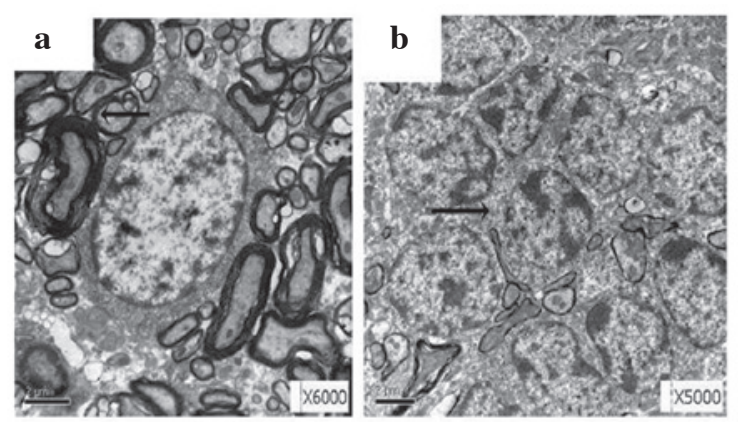

B
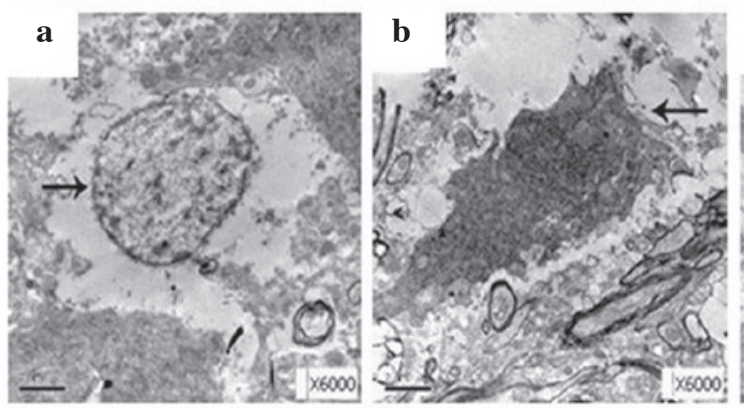

C
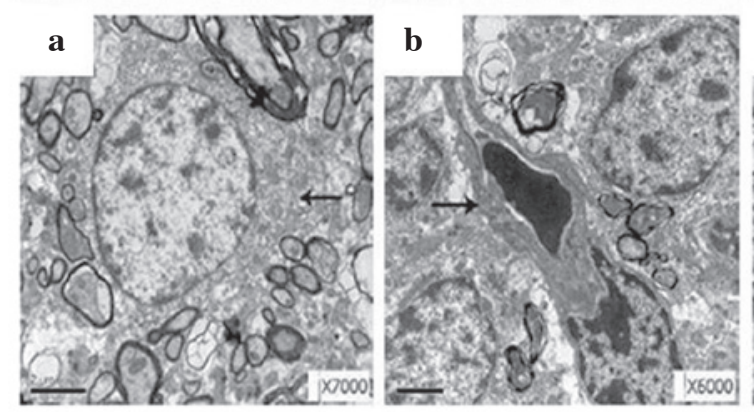
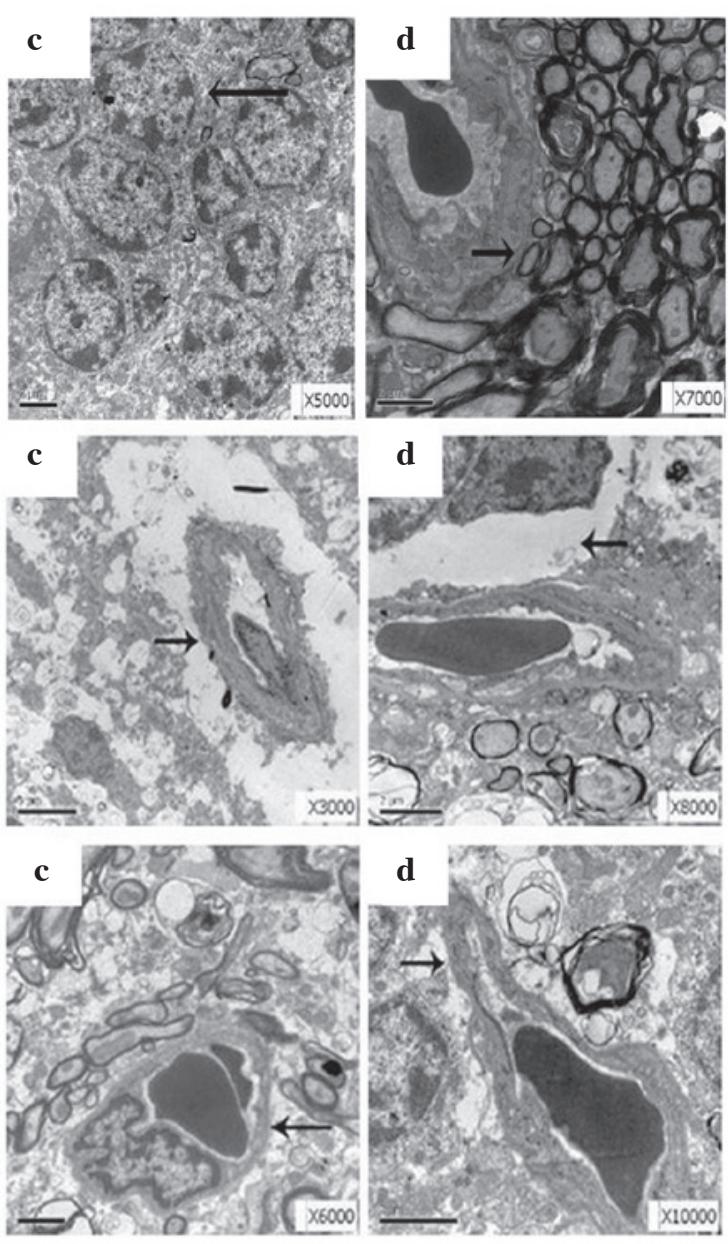

Figure 2. Electron microscopy analysis of the ultrastructure alterations in rat brain tissues derived from the various treatment groups. At least five fields with positive results were identified in each case. (A) Brain tissue ultrastructure of the control group, showing (a) normal neurons, (b and c) neuroglial cells, and (d) capillary structure. (B) Brain tissue ultrastructure of the hypoxic-hypercapnia group, showing (a) neural edema, (b) neuronal cell apoptosis, (c) endothelial cells protruding towards the cavity, and (d) regions of swelling surrounding the capillaries. (C) Brain tissue ultrastructure of the curcumin group, showing reduced levels of edema in ( $a$ and b) the neurons and (c and d) in the area surrounding the capillaries. Arrows indicate (a) neuron, (b and c) neuroglial cells or endothelial cells, and (d) surrounding capillaries.

scriptional abundance value of the AQP4 gene was expressed as $2^{-\Delta \Delta \mathrm{Cq}}(26)$.

Statistical analysis. SPSS 17.0 statistical software (SPSS Inc., Chicago, IL, USA) was applied for the processing and analyzing of all data. Data are presented as the mean \pm standard error of the mean. Normality testing was performed and Student-Newman-Keuls-q tests were conducted for the examination of the overall mean of the samples. Variance homogeneity tests were used in order to compare the mean of multiple-sample groups. In order analyze the variance between two groups, one-way analysis of variance was conducted. $\mathrm{P}<0.05$ was considered to indicate a statistically significant difference.

\section{Results}

Morphological observations using light microscopy. Morphological characteristics of the rat brain tissue samples from the CK, HH, CU,DMSO and GM1 groups were investigated under a light microscope (Fig. 1). In the CK group, HE staining demonstrated that the area surrounding the brain capillaries was slightly widened and the nerve cells were arranged neatly. In addition, normal cell borders, nuclei, nucleoli and cell extensions were observed. Conversely, in the $\mathrm{HH}$ group tissue samples, signs of edema were detected, including a condensed cytoplasm, shrunken membrane, dwindled cell body and pyknotic or fragmented nuclei. Furthermore, HE staining demonstrated that the area surrounding the nerve cells was widened, and the nerve cells were shrunken. Cells in the brain tissue of the DMSO group rats were similar to those of the $\mathrm{HH}$ group. Conversely, CU treatment markedly attenuated edema in the brain. In the $\mathrm{CU}$ group tissues, the brain cells were arranged neatly and the area surrounding the capillaries was only slightly expanded. In addition, vascular endothelial cells and astrocytes surrounding the capillaries did not exhibit obvious swelling. The morphological characteristics observed in the brain of the GM1 group rats were similar to those detected for the $\mathrm{CU}$ group.

Comparison of the ultrastructures of the brain tissue samples from the $\mathrm{CK}, \mathrm{HH}$ and $\mathrm{CU}$ groups. The ultrastructures of the rat brain tissue samples derived from the $\mathrm{CK}, \mathrm{HH}$ and $\mathrm{CU}$ groups were investigated using electron microscopy. In the CK group, the nuclei of neuronal cells appeared large and round, and the nucleolus exhibited a clear boundary with 
double-layer envelopes. In addition, the nuclei of the astrocytes appeared normal and their extensions did not exhibit swelling. No edema was detected in the area surrounding the capillaries and intraluminal red cells, and the endothelial cells were closely interconnected (Fig. 2A). Conversely, in the $\mathrm{HH}$ group, the neurons were markedly swollen, and contained enlarged rough endoplasmic reticuli and swollen mitochondria. Furthermore, some neurons and astrocytes exhibited signs of apoptosis, including nuclear envelope shrinkage and nuclear pyknosis, the capillaries were shrunken and deformed, and the endothelial cells protruded towards the cavity and the interconnections of the endothelium were enlarged. The extension of the astrocytes in the blood-brain barrier (BBB) swelled and vesicles were formed (Fig. 2B). CU treatment markedly attenuated HHBD-induced ultrastructure destruction in the brain tissue, as demonstrated by marked improvements in ultrastructural integrity. In addition, the number of cells exhibiting nuclear envelope shrinkage and nuclear pyknosis were markedly decreased, and edema of the neurons, astrocytes and the area surrounding the capillaries was improved (Fig. 2C).

Water content measurement. Water content in the brain tissues from the various treatment groups was analyzed. Water levels in the $\mathrm{HH}$ group rats were significantly increased, as compared with the CK group ( $\mathrm{P}<0.05$; Fig. 3). No significant differences were detected between the $\mathrm{HH}$ and DMSO groups $(\mathrm{P}>0.05$; Fig. 3). Conversely, water levels in the CU group were significantly decreased, as compared with the HH group $(\mathrm{P}<0.05$; Fig. 3). Notably, GM1 treatment had the same effects as CU treatment (P>0.05; Fig. 3).

SABC immunohistochemical analysis of AQP4 expression levels. In order to investigate the underlying mechanisms of HHBD, the present study investigated the protein expression levels of AQP4 in the brain cortex, cerebellum and brainstem tissue samples from the various groups using the SABC immunohistochemical method (Fig. 4). In addition, optical density (OD) values of the AQP4 staining were quantified and compared (Table I). AQP4 protein expression levels were negligible in the brain cortex of the CK group, as demonstrated by the minor AQP4 staining observed in the cell membranes and axons. Conversely, elevated AQP4 protein expression levels were detected in the $\mathrm{HH}$ group, as demonstrated by dark brown staining of the cell membranes and axons along the capillaries of the $\mathrm{HH}$ group tissue samples. Furthermore, the OD value of AQP4 staining in the $\mathrm{HH}$ group was significantly increased, as compared with the $\mathrm{CK}$ group $(\mathrm{P}<0.05$; Table I). Increased AQP4 staining and higher OD values were similarly detected in brain tissue samples from the DMSO-treated rats. However, AQP4 staining was significantly decreased in the brain cortex of the CU and GM1 groups, as compared with the HH group $(\mathrm{P}<0.05$; Table I). A similar phenomenon was detected in the cerebellum and brainstem tissues. Increased AQP4 staining and higher OD values were detected for the $\mathrm{HH}$ and DMSO groups, as compared with the CK, CU and GM1 groups; thus suggesting that $\mathrm{CU}$ treatment is able to significantly decrease HHBD-induced AQP-4 expression in various rat brain tissues.

Western blot analysis of the AQP4 protein expression. Western blot analysis was conducted in order to further

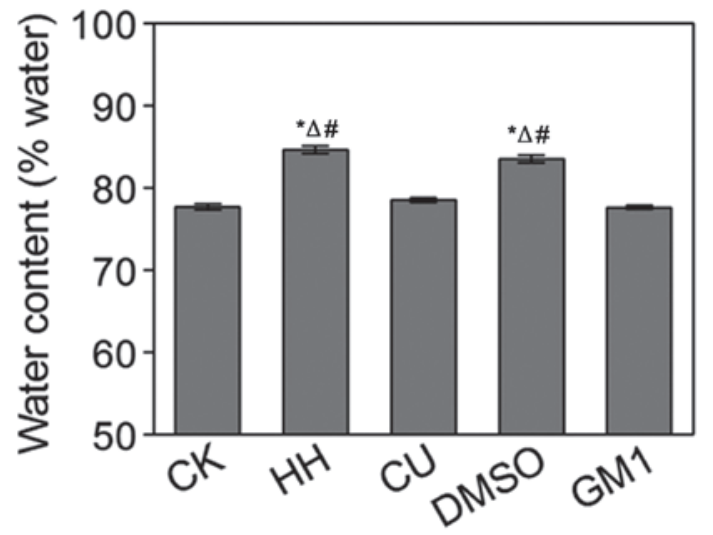

Figure 3. Water content of the rat brain tissue derived from the various treatment groups. Data are presented as the mean \pm standard error of the mean. ${ }^{*} \mathrm{P}<0.05$ vs. the $\mathrm{CK}$ group; ${ }^{\Delta} \mathrm{P}<0.05$ vs. the $\mathrm{CU}$ group; ${ }^{\#} \mathrm{P}<0.05$ vs. the GM1 group. CK, control group; $\mathrm{HH}$, hypoxia-hypercapnia group; $\mathrm{CU}$, curcumin group; DMSO, dimethyl sulfoxide solvent group; GM1, monosialoganglioside group.

investigate alterations in the protein expression levels of AQP4 in the brain tissues derived from the various treatment groups. The protein expression levels of AQP4 in the $\mathrm{HH}$ and DMSO groups were significantly increased ( $\mathrm{P}<0.05$; Fig. 5$)$, as compared with the CK group. However, CU treatment inhibited the HHBD-induced increase in AQP4 protein expression levels in the CU group, as compared with the HH group. In addition, GM1 treatment similarly inhibited AQP4 expression.

AQP4 gene expression analysis. The mRNA expression levels of AQP-4 in the various treatment groups were detected by RT-qPCR. The mRNA expression levels of AQP-4 were increased by 30 -fold in the $\mathrm{HH}$ group and 28 -fold in the DMSO group, as compared with the CK group. Conversely, in the CU and GM1 groups, the mRNA expression levels of AQP-4 were increased by 3.7-fold and 2.0-fold, as compared with the CK group, respectively. These results were consistent with those of the SABC immunohistochemical and western blot analyses (Fig. 6).

\section{Discussion}

HHBD, which is a common result of asphyxiation and COPD, is detrimental to the nervous system and has previously been associated with cognitive dysfunction, nervous system disorders and mental retardation (27). In addition, hypoxic brain damage accounts for the majority of injuries observed in forensic cases (28). Therefore, analyzing the mechanisms underlying HHBD, and identifying novel therapeutic drugs for the treatment of hypoxic brain injury, are of great value in clinical treatment and forensic pathology.

Previous studies have demonstrated that various traditional Chinese medicines exert neuroprotective effects that may be used to treat patients with nerve injuries $(11,29)$. Our previous study suggested that $\mathrm{CU}$ was able to alleviate HIBD-induced brain edema in a rat model of HIBD (18), and the present study investigated the effects of $\mathrm{CU}$ in a rat model of HHBD. In the $\mathrm{HH}$ group, neurons appeared markedly swollen, organelles were disintegrated, and cells 


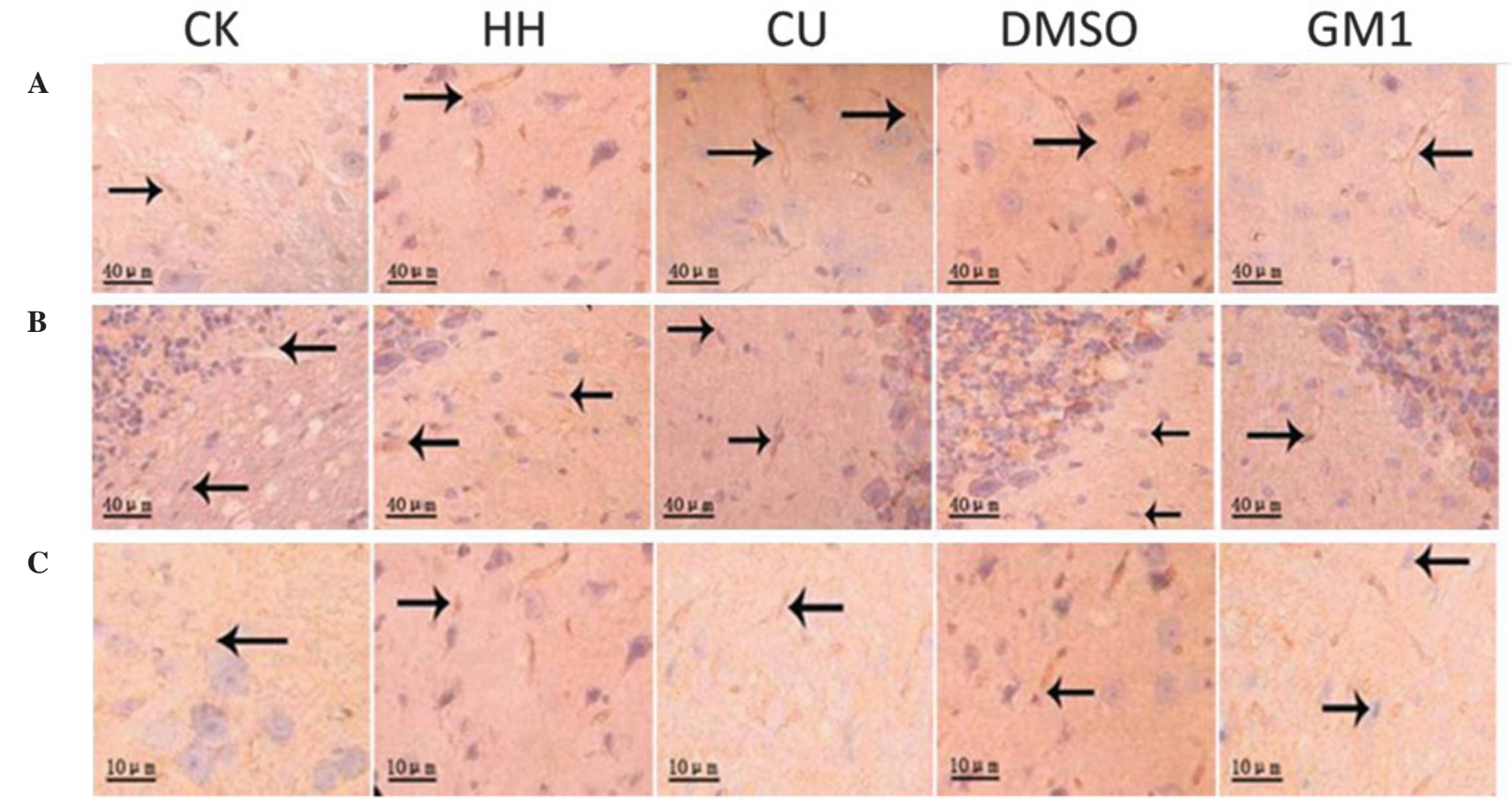

Figure 4. Streptavidin-biotin complex immunohistochemical analysis of AQP4 protein expression levels in the (A) brain cortex, (B) cerebellum and (C) brainstem of the various groups (3,3'-diaminobenzidine; magnification, x100; 14 days). At least five fields with positive results were identified in each case. Arrows indicate AQP4-positive cells and dark brown indicates strongly positive AQP4 staining. AQP4, aquaporin-4; CK, control group; HH, hypoxia-hypercapnia group; CU, curcumin group; DMSO, dimethyl sulfoxide solvent group; GM1, monosialoganglioside group.

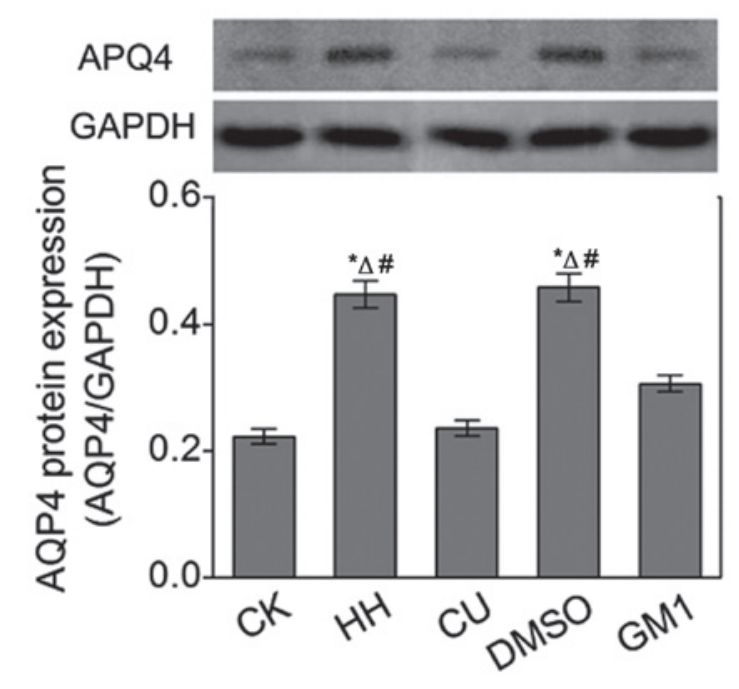

Figure 5. AQP4 protein expression levels in the rat brain tissues were analyzed using western blotting and OD values were calculated. GAPDH was used as an internal control. Data are presented as the mean \pm standard error of the mean. ${ }^{*} \mathrm{P}<0.05$ vs. the $\mathrm{CK}$ group; ${ }^{\Delta} \mathrm{P}<0.05$ vs. the $\mathrm{CU}$ group; ${ }^{\#} \mathrm{P}<0.05$ vs. the GM1 group. AQP4, aquaporin-4; OD, optical density; CK, control group; $\mathrm{HH}$ hypoxia-hypercapnia group; $\mathrm{CU}$, curcumin group; DMSO, dimethyl sulfoxide solvent group; GM1, monosialoganglioside. group; GAPDH, glyceraldehyde 3-phosphate dehydrogenase.

exhibited signs of apoptosis. In addition, the water content in the $\mathrm{HH}$ group cells was significantly higher, as compared with the CK group cells. These results suggested that $\mathrm{HH}$ conditions induced brain edema and destroyed brain tissue and cell structures in the rats, which is consistent with a previous report (30).

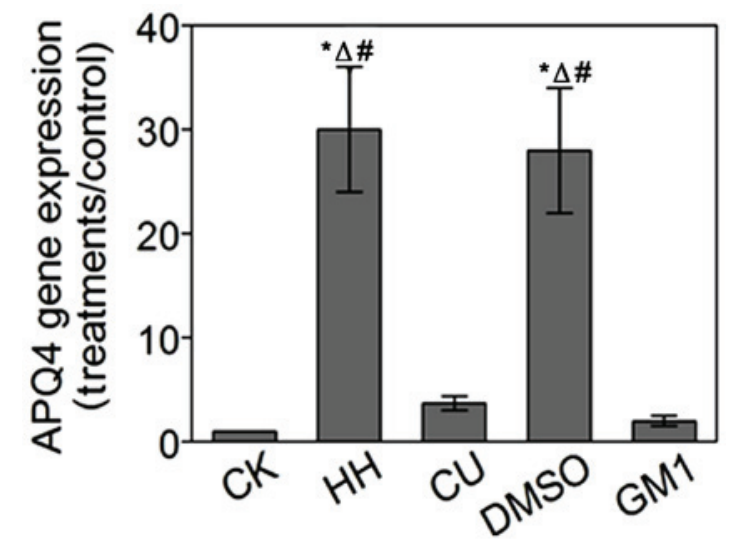

Figure 6. mRNA expression levels of AQP4 in rat brain tissue derived from the various groups were analyzed by reverse transcription-quantitative polymerase chain reaction. Data are presented as the mean \pm standard error of the mean. ${ }^{*} \mathrm{P}<0.05$ vs. the $\mathrm{CK}$ group; ${ }^{\Delta} \mathrm{P}<0.05$ vs. the $\mathrm{CU}$ group; ${ }^{*} \mathrm{P}<0.05$ vs. the GM1 group. AQP4, aquaporin-4; $\mathrm{CK}$, control group; $\mathrm{HH}$, hypoxia-hypercapnia group; CU, curcumin group; DMSO, dimethyl sulfoxide solvent group; GM1, monosialoganglioside group.

In the present study, CU treatment alleviated the $\mathrm{HH}$-induced alterations observed in the HH group; thus suggesting that $\mathrm{CU}$ was able to attenuate HHBD-induced brain edema. Dohare et al (31) reported that CU treatment decreased edema formation in a rat model of cerebral thromboembolism in a dose-dependent manner. Similarly, Ghoneim et al (32) demonstrated that $\mathrm{CU}$ treatment attenuated ischemic-induced brain edema in the rat forebrain, and King et al (33) reported that $\mathrm{CU}$ treatment significantly decreased the water content and relieved brain edema in a rat model of intracerebral hemor- 
Table I. Detection of AQP4 protein expression levels in brain tissues of the various treatment groups by SABC immunohistochemistry (OD values; $n=6$ ).

\begin{tabular}{|c|c|c|c|c|c|}
\hline Region & CK group & HH group & CU group & DMSO group & GM1 group \\
\hline Cerebrum & $0.23 \pm 0.02$ & $0.47 \pm 0.01^{\mathrm{a}, \mathrm{b}}$ & $0.36 \pm 0.02$ & $0.48 \pm 0.02^{\mathrm{a}, \mathrm{b}}$ & $0.36 \pm 0.02$ \\
\hline Cerebellum & $0.24 \pm 0.03$ & $0.48 \pm 0.02^{\mathrm{a}, \mathrm{b}}$ & $0.38 \pm 0.02$ & $0.49 \pm 0.02^{\mathrm{a}, \mathrm{b}}$ & $0.38 \pm 0.03$ \\
\hline Brainstem & $0.22 \pm 0.03$ & $0.40 \pm 0.03^{\mathrm{a}, \mathrm{b}}$ & $0.30 \pm 0.03$ & $0.40 \pm 0.02^{\mathrm{a}, \mathrm{b}}$ & $0.29 \pm 0.03$ \\
\hline
\end{tabular}

Data are presented as the mean \pm standard error of the mean. ${ }^{a} \mathrm{P}<0.05$ vs. the $\mathrm{CK}$ group; ${ }^{\mathrm{b}} \mathrm{P}<0.05$ vs. the $\mathrm{CU}$ and GM1 groups. AQP4, aquaporin-4; SABC, streptavidin-biotin complex; OD, optical density; CK, control group; CU, curcumin-treated group; HH, hypoxia-hypercapnia group; DMSO, dimethyl sulfoxide solvent group; GM1, monosialoganglioside group.

rhage. Despite differences in these rat models and the brain tissues used, CU treatment was able to attenuate brain edema in all cases (31-33); however, the mechanisms underlying CU-mediated attenuation of brain edema are yet to be defined thoroughly, and the precise mechanisms may have differed among the various models.

AQPs are selective water channels that reside on the cell membrane. A total of six AQPs have previously been identified in mammalian brain tissues, of which AQP1 and AQP4 are the mostly widely distributed in the central nervous system. It has previously been demonstrated that AQP4, which is located on both sides of the $\mathrm{BBB}$, is primarily expressed in the end-feet of astrocytes and in capillary endothelial cells. AQP4 may have an important role in aiding the passage of water through the $\mathrm{BBB}$, and therefore upregulation of AQP4 may be a molecular mechanism underlying brain edema $(34,35)$.

The results of the present study suggested that CU was able to attenuate chronic hypoxia-induced cerebral damage by regulating AQP4 expression levels. SABC immunohistochemical analysis detected significantly increased AQP4 protein expression levels in the brain cortex, cerebellum and brainstem of rats in the $\mathrm{HH}$ group, as compared with the $\mathrm{CK}$ group. However, the CU-treated rats exhibited decreased AQP4 protein expression levels, as compared with the $\mathrm{HH}$ group. Our previous study demonstrated that $\mathrm{CU}$ was able to decrease the expression levels of AQP4 in the hippocampus of a rat model of HIBD (18). In the present study, CU treatment was able to reduce the AQP4 expression levels in various other brain tissues following chronic HH. Furthermore, western blotting and RT-qPCR were conducted in order to thoroughly investigate the AQP4 protein and mRNA expression levels, respectively. As expected, the mRNA and protein expression levels of AQP4 were increased in the $\mathrm{HH}$ group brain tissues, whereas $\mathrm{CU}$ treatment was able to inhibit this elevation, and this was consistent with the SABC imunohistochemistry results.

Laird et al (36) reported that pre- or post-treatment with CU significantly alleviated head trauma-induced brain edema in mice, and these protective effects were associated with significantly decreased acute peri-contusional expression of interleukin-1B and AQP4. In addition, Ji et al (37) demonstrated that chemically synthesized CU was able to inhibit the expression of AQP4 and the phosphorylation of c-Jun $\mathrm{N}$-terminal kinase, in order to protect the structure of the BBB and alleviate cerebral edema following focal cerebral ischemia-reperfusion damage in rats. Furthermore, CU was shown to attenuate edema in other nerve tissues, including spinal cord tissue, and this was associated with the inhibition of AQP4 overexpression and the Janus kinase/signal transducers and activators of transcription signaling pathway (38).

In the present study, downregulation of HHBD-induced AQP4 expression had an important role in the CU-mediated attenuation of brain edema. However, the signaling pathway underlying CU-regulated AQP4 expression is currently unclear. Previous studies have suggested that the brain edema-induced upregulation of AQP4 expression may be associated with decreased $\mathrm{Na}^{+}-\mathrm{K}^{+}$-adenosine triphosphatase (ATPase) activity and dysfunctional $\mathrm{Na}^{+}$influx/ $\mathrm{K}^{+}$efflux. Cerebral hypoxia and ischemia decreased the activity of $\mathrm{Na}^{+}-\mathrm{K}^{+}$-ATPase, which was associated with an imbalance in the osmotic pressure on both sides of the cell membrane, activation of osmotic pressure sensors, upregulation of AQP4 expression and signs of edema $(39,40)$. Therefore, $\mathrm{Na}^{+}-\mathrm{K}^{+}$-ATPase may be an intermediate component of the CU-regulated AQP4 expression signal transduction pathway; however further experiments are required to investigate this.

The present study investigated the effects of GM1 against HHBD-induced brain edema. GM1 is a widely recognized chemical that exerts neuroprotective effects (41). The results of the present study suggested that GM1 and CU treatment were able to alleviate HHBD-induced brain edema and AQP4 expression. GM1 has previously been shown to maintain the activities of the $\mathrm{Na}^{+}-\mathrm{K}^{+}-\mathrm{ATP}$ and $\mathrm{Ca}^{2+}-\mathrm{Mg}^{2+}$-ATP enzymes in the membrane of central nerve cells, which in turn maintained the intracellular ion balance, attenuated edema of nerve cells and prevented the accumulation of $\mathrm{Ca}^{2+}$ in the cells (42). Therefore, CU and GM1 may share a common signaling pathway in their regulation of AQP4 expression; however further research is required in order to confirm this.

In conclusion, the results of the present study suggested that CU may alleviate chronic $\mathrm{HH}$-induced brain edema in a rat model of HHBD by inhibiting HHBD-induced upregulation of AQP4. Therefore, CU may be considered a potential therapeutic drug for the treatment of patients with chronic HHBD. Further experiments, in particular clinical experiments, are required in order to evaluate the potential clinical application of CU.

\section{Acknowledgements}

The present study was supported by the Shanghai Key Lab of Forensic Medicine (grant no. KF1403); the Natural Science 
Foundation of Zhejiang Province (grant no. LQ13H150002); and the Wenzhou Municipal Science and Technology Bureau Science and Technology Planning Project (grant no. Y20100185).

\section{References}

1. Bakay L and Lee JC: The effect of acute hypoxia and hypercapnia on the ultrastructure of the central nervous system. Brain 91 697-706, 1968

2. Yu L, Yi J, Ye G, Zheng Y, Song Z, Yang Y, Song Y, Wang Z and Bao Q: Effects of curcumin on levels of nitric oxide synthase and AQP-4 in a rat model of hypoxia-ischemic brain damage. Brain Res 1475: 88-95, 2012.

3. Ainslie PN and Ogoh S: Regulation of cerebral blood flow in mammals during chronic hypoxia: A matter of balance. Exp Physiol 95: 251-262,2010.

4. Donkin JJ and Vink R: Mechanisms of cerebral edema in traumatic brain injury: Therapeutic developments. Curr Opin Neurol 23: 293-299, 2010.

5. Von Sarnowski B, Kleist-Welch Guerra W, Kohlmann T, Moock J, Khaw AV, Kessler C, Schminke U and Schroeder HW: Long-term health-related quality of life after decompressive hemicraniectomy in stroke patients with life-threatening space-occupying brain edema. Clin Neurol Neurosurg 114: 627-633, 2012

6. Rite I, Machado A, Cano J and Venero JL: Intracerebral VEGF injection highly upregulates AQP4 mRNA and protein in the perivascular space and glia limitans externa. Neurochem Int 52: 897-903, 2008.

7. Yoshida H, Yanai H, Namiki Y, Fukatsu-Sasaki K, Furutani N and Tada N: Neuroprotective effects of edaravone: A novel free radical scavenger in cerebrovascular injury. CNS Drug Rev 12: 9-20, 2006 .

8. Mcconeghy KW, Hatton J, Hughes L and Cook AM: A review of neuroprotection pharmacology and therapies in patients with acute traumatic brain injury. CNS Drugs 26: 613-636, 2012.

9. Gaire BP and Kim H: Neuroprotective effects of Fructus Chebulae extracts on experimental models of cerebral ischemia. J Tradit Chin Med 34: 69-75, 2014.

10. Liu QS, Chen XY, Zhuang SJ and Li KQ: Research on effect of Baimai powder effective compounds group promotes neurogenesis and maintains of neural stem cells after cerebral infarction. Zhongguo Zhong Yao Za Zhi 38: 3776-3781, 2013 (In Chinese)

11. Gupta YK, Briyal S and Gulati A: Therapeutic potential of herbal drugs in cerebral ischemia. Indian J Physiol Pharmacol 54: 99-122, 2010.

12. Bu Y, Lee K, Jung HS and Moon SK: Therapeutic effects of traditional herbal medicine on cerebral ischemia: A perspective of vascular protection. Chin J Integr Med 19: 804-814, 2013.

13. Zhou H, Beevers CS and Huang S: Targets of curcumin. Current drug targets 12: 332-347, 2011.

14. Wilken R, Veena MS, Wang MB and Srivatsan ES: Curcumin A review of anti-cancer properties and therapeutic activity in head and neck squamous cell carcinoma. Mol Cancer 10: 12, 2011.

15. Chen SY, Chen Y, Li YP, Chen SH, Tan JH, Ou TM, Gu LQ and Huang ZS: Design, synthesis and biological evaluation of curcumin analogues as multifunctional agents for the treatment of Alzheimer's disease. Bioorg Med Chem 19: 5596-5604, 2011.

16. Tu XK, Yang WZ, Chen JP, Chen Y, Ouyang LQ, Xu YC and Shi SS: Curcumin inhibits TLR2/4-NF- $\kappa$ B signaling pathway and attenuates brain damage in permanent focal cerebral ischemia in rats. Inflammation 37: 1544-1551, 2014.

17. Kuo CP, Lu CH, Wen LL, Cherng CH, Wong CS, Borel CO, Ju DT, Chen CM and Wu CT: Neuroprotective effect of curcumin in an experimental rat model of subarachnoid hemorrhage. Anesthesiology 115: 1229-1238, 2011.

18. Yu L, Yi J, Ye G, Zheng Y, Song Z, Yang Y, Song Y, Wang Z and Bao Q: Effects of curcumin on levels of nitric oxide synthase and AQP-4 in a rat model of hypoxia-ischemic brain damage. Brain Res 1475: 88-95, 2012.

19. Yu L, Yi J, Ye G, Zheng Y, Song Z, Yang Y, Song Y, Wang Z and Bao Q: Effects of curcumin on levels of nitric oxide synthase and AQP-4 in a rat model of hypoxia-ischemic brain damage. Brain Res 1475: 88-95, 2012.

20. Wang Hua LH: Preservation of animal tissue within the RNA method to improve. Sheng Wu Ji Shu Tong Bao 4, 2010 (In Chinese).
21. Chen FH, Wang L and Hu LH: Real time fluorescent quantitative RT-PCR reference genes selection. Zhong Guo Lin Chuang Jian Yan Za Zhi 23: 393-395, 2005 (In Chinese).

22. Shibata M, Yamawaki T, Sasaki T, Hattori H, Hamada J, Fukuuchi Y, Okano H and Miura M: Upregulation of Akt phosphorylation at the early stage of middle cerebral artery occlusion in mice. Brain Res 942: 1-10, 2002.

23. Beziaud T, Chen XR, El Shafey N, Fréchou M, Teng F, Palmier B, Beray-Berthat V, Soustrat M, Margaill I, Plotkine M, et al: Simvastatin in traumatic brain injury: Effect on brain edema mechanisms. Crit Care Med 39: 2300-2307, 2011.

24. Sun L, Yang L, Xu YW, Liang H, Han J, Zhao RJ and Cheng Y: Neuroprotection of hydroxysafflor yellow A in the transient focal ischemia: Inhibition of protein oxidation/nitration, 12/15-lipoxygenase and blood-brain barrier disruption. Brain Res 1473: 227-235, 2012.

25. Tang Y, Cai D and Chen Y: Thrombin inhibits aquaporin 4 expression through protein kinase $\mathrm{C}$-dependent pathway in cultured astrocytes. J Mol Neurosci 31: 83-93, 2007.

26. Livak KJ and Schmittgen TD: Analysis of relative gene expression data using real-time quantitative PCR and the 2(-Delta Delta C(T)) method. Methods 25: 402-408, 2001.

27. Zheng GQ, Wang Y and Wang XT: Chronic hypoxia-hypercapnia influences cognitive function: A possible new model of cognitive dysfunction in chronic obstructive pulmonary disease. Med Hypotheses 71: 111-113, 2008.

28. Oechmichen M and Meissner C: Cerebral hypoxia and ischemia: The forensic point of view: A review. J Forensic Sci 51: 880-887, 2006.

29. Chen YF: Traditional Chinese herbal medicine and cerebral ischemia. Front Biosci (Elite Ed) 4: 809-817, 2012

30. Jeremitsky E, Omert L, Dunham CM, Protetch J and Rodriguez A: Harbingers of poor outcome the day after severe brain injury: Hypothermia, hypoxia and hypoperfusion. J Trauma 54: 312-319, 2003.

31. Dohare P, Garg P, Jain V, Nath C and Ray M: Dose dependence and therapeutic window for the neuroprotective effects of curcumin in thromboembolic model of rat. Behav Brain Res 193: 289-297, 2008

32. Ghoneim AI, Abdel-Naim AB, Khalifa AE and El-Denshary ES: Protective effects of curcumin against ischaemia/reperfusion insult in rat forebrain. Pharmacol Res 46: 273-279, 2002.

33. King MD, Mccracken DJ, Wade FM, Meiler SE, Alleyne CH Jr and Dhandapani KM: Attenuation of hematoma size and neurological injury with curcumin following intracerebral hemorrhage in mice. J Neurosurg 115: 116-123, 2011.

34. Papadopoulos MC and Verkman AS: Aquaporin-4 and brain edema. Pediatr Nephrol 22: 778-784, 2007.

35. Loreto $\mathrm{C}$ and Reggio E: Aquaporin and vascular diseases. Curr Neuropharmacol 8: 105-111, 2010.

36. Laird MD, Sukumari-Ramesh S, Swift AE, Meiler SE, Vender JR and Dhandapani KM: Curcumin attenuates cerebral edema following traumatic brain injury in mice: A possible role for aquaporin-4? J Neurochem 113: 637-648, 2010.

37. Ji FT, Cao MH, Liang JJ, Liu L, Li F and Bu XZ: Effects of chemical synthesized curcumin preconditioning on the expression of AQP 4 and cerebral edema after focal cerebral ischemia/reperfusion damage in rats. Zhong Guo Yao Li Xue Tong Bao 4: 019, 2011 (In Chinese).

38. Zu J, Wang Y, Xu G, Zhuang J, Gong $\mathrm{H}$ and Yan J: Curcumin improves the recovery of motor function and reduces spinal cord edema in a rat acute spinal cord injury model by inhibiting the JAK/STAT signaling pathway. Acta Histochem 116: 1331-1336, 2014.

39. Moftakhar P, Lynch MD, Pomakian JL and Vinters HV: Aquaporin expression in the brains of patients with or without cerebral amyloid angiopathy. J Neuropathol Exp Neurol 69: 1201-1209, 2010

40. Yang B, Zador Z and Verkman A: Glial cell aquaporin-4 overexpression in transgenic mice accelerates cytotoxic brain swelling. J Biol Chem 283: 15280-15286, 2008.

41. Vieira KP, De Almeida E Silva Lima, Zollner AR, Malaguti C, Vilella CA and De Lima Zollner R: Ganglioside GM1 effects on the expression of nerve growth factor (NGF), Trk-A receptor, proinflammatory cytokines and on autoimmune diabetes onset in non-obese diabetic (NOD) mice. Cytokine 42: 92-104, 2008.

42. Chen ZG, Lu YC, Zhu C, Zhang GJ, Ding XH and Jiang JY: Effects of ganglioside GM1 on reduction of brain edema and amelioration of cerebral metabolism after traumatic brain injury. Chin J Traumatol 6: 23-27, 2003. 